\title{
Estado nutricional y aspectos familiares en niños peruanos con VIH en la era TARAA
}

\section{Nutritional Status and Family issues in Children Peruvian with HIV in the HAART}

\author{
Edwin Miranda ${ }^{1}$, Sonia Farfán ${ }^{2}$, Sara Barrientos ${ }^{3}$, Lizzet Lara ${ }^{4}$, \\ Doris $\mathrm{Coz}^{5}$
}

http://dx.doi.org/10.21503/CienciayDesarrollo.2013.v16i1.08

\section{RESUMEN}

Objetivo: Describir el estado nutricional y aspectos familiares en niños con VIH en la era TARAA, en un país en estadio de epidemia concentrada del VIH con acceso gratuito a tratamiento antirretroviral.

Material y Métodos. Estudio retrospectivo se revisaron historias clínicas, estudiamos a los niños con infección de VIH, por trasmisión vertical, que iniciaron TARAA entre 2007 a 2009 en un hospital pediátrico de Perú. Se comparó con un grupo control no infectados VIH para determinar el riesgo de desnutrición.

Resultados: De los 75 niños enrolados, 34 (45\%) fueron hombres, el promedio de edad de inicio de TARAA fue 3,6 años, la mediana de carga viral de $\log 5,14$ copias $/ \mathrm{ml}$. Los niños con inmunodepresión severa (CD4<25\%) fue el 68,0\% (51/75), el 15\% ha sufrido la pérdida de algún padre, el 36\% recibe el cuidado de algún miembro de la familia diferente a los padres o viven en orfanatos, el $20 \%$ son hijos de madres solteras y el porcentaje de los niños con VIH con algún grado de desnutrición es de 69,3\%, siendo el peso bajo para la edad más frecuente con el 66,7\%, esto resulta un OR de 6,2 y 10,5 respectivamente.

Conclusión: Existe un riesgo elevado de déficit de estado nutricional especialmente en el peso para la edad en los niños con VIH al momento de su diagnóstico. Muchos niños con VIH presentan nuevas formas de convivencias bajo el cuidado de sus familiares u orfanatos que lo hacen propensos a la dificultad en la inserción social.

Palabras Claves: $H I V$, niños, TARAA

\section{ABSTRACT}

Objective: To describe the nutritional status and family issues in children with HIV in the HAART era in a country stage concentrated HIV epidemic with free access to antiretroviral treatment.

Material and Methods: We examined a retrospective study of medical charts and studied children with HIV infection by vertical transmission who initiated HAART between 2007 and 2009 at a Paediatric Hospital in Peru. It was compared with HIV-uninfected control group to determine the risk of malnutrition.

Results: Of the 75 children, 34 (45\%) were male, the average age of onset of HAART was 3.6 years, the median viral load of $5.14 \log$ copies $/ \mathrm{ml}$, children with severe immunodeficient $(\mathrm{CD} 4<25 \%)$ was

\footnotetext{
1 Instituto Nacional de Salud del Niño, Lima, Perú. Docente de la EAP Medicina Humana de la UAP. Médico Infectólogo. e-mail: emirandach@hotmail.com

2 Hospital Nacional 2 de Mayo. Lima, Perú. Magister en Salud Pública y Comunitaria. Enfermera especialista en Cuidados Intensivos. soniaf42@hotmail.com

3 Instituto Nacional de Salud del Niño, Lima, Perú. Enfermera, especialista en Enfermería Pediátrica. sara_barrientos2005@hotmail.com

4 Hospital Emergencias Pediátricas. Lima, Perú. Enfermera especialista en Cuidados Intensivos.

5 Hospital Nacional 2 de mayo. Lima, Perú. Enfermera, especialista en Enfermería Pediátrica. Doriscozb1@hotmail.com
} 
$68.0 \%(51 / 75), 15 \%$ have experienced the loss of a parent, $36 \%$ receive care from a family member other than the parents or living in orphanages, $20 \%$ are born to mothers single and the percentage of children with HIV with some degree of malnutrition is $69.3 \%$, the weight-for-age more frequent with $66.7 \%$, this is an OR of 6.2 and 10.5 respectively.

Conclusion: There is a high risk of nutritional deficiency especially in the weight for age in children with HIV at the time of diagnosis. Many children with HIV are new forms of coexistence under the care of relatives or orphanages that make it prone to difficulty in social inclusion.

Keywords: HIV, children, HAART

\section{INTRODUCCIÓN}

La trasmisión del VIH en las familias mayormente se inicia con la infección del padre que contrae la infección al presentar relaciones extramatrimoniales, posteriormente la madre es infectada por VIH a través de contactos heterosexuales con su pareja infectada, si la relación culmina en embarazo los hijos son infectados verticalmente por la madre. En la era TARAA (tratamiento antirretroviral altamente activo) el acceso a los antirretrovirales durante el embarazo y el parto son medidas adecuadas para disminuir dicha transmisión, llegando disminuir de 30\% a 10\%, $\mathrm{y}$ en algunos casos puede disminuir a menos del $2 \%$, particularmente en las zonas de recursos limitados $^{1,2}$.

En Estados Unidos aparecen los primeros casos confirmados de VIH en niños en 1982. Siendo el primer caso de VIH en adultos en el Perú en $1983^{3}$ y el primer caso de VIH en niños por trasmisión vertical fue en 1990 según la oficina de epidemiología del INSN.

El Perú presenta un programa de prevención y control del VIH/SIDA que incluye estrategias de prevención de la transmisión vertical y tratamiento antirretroviral para los casos de VIH desde mayo 2004, siendo el INSN la institución que atiende el mayor número de niños infectados con VIH en el Perú .

Las enfermeras en los hospitales aparte de labores asistenciales, también cumplen una función social, como son la atención de las familias de los pacientes por lo que es necesario conocer los aspectos sociales y nutricionales de una población considerada como vulnerable como son los niños de VIH; información no disponible en nuestro medio por lo que decidimos realizar el presente estudio con el objetivo de describir los aspectos familiares y de estado nutricional en niños con infección de VIH en la era TARAA, en un país en vías de desarrollo con patrón epidemiológico de estadio de epidemia concentrada del VIH que brinda tratamiento antirretroviral gratuito, con la finalidad de apoyar en el conocimiento de los niños que viven con VIH para contribuir a la formulación de estrategias adecuadas.

\section{MATERIALES Y MÉTODOS}

\section{Pacientes y población}

Realizamos un estudio descriptivo retrospectivo, enrolamos a niños menores de 14 años de edad con diagnóstico de VIH, trasmitidos verticalmente, que inician TARAA entre 2007 y 2009, atendidos en el INSN (Instituto Nacional del Niño). Siendo centro de referencia nacional revisamos las historias clínicas y verificamos las pruebas de laboratorio del diagnóstico de VIH mediante PCR ADN - VIH - 1 o ELISA (detección de anticuerpos totales) para VIH, así como linfocitos CD4 (CD4) y carga viral (CV). Fueron excluidos aquellos que presentaban información incompleta en las historias clínicas, si fueron transferidos, iniciaron TARAA en otra institución. Se calculó el porcentaje de CD4 
usando el valor absoluto dividido por el valor linfocitos totales de sangre periférica por $\mathrm{ml}$, el cual se usó para la evaluación del estado inmunológico según las recomendaciones de WHO y Centers for Disease Control and Prevention de la siguiente manera: Inmunosupresión severa para $\leq 11$ meses, $\mathrm{si}<25 \%$ o $<1500 \mathrm{cel} / \mathrm{mm} ; 12$ meses a 35 meses $<20 \%$ o $<750 \mathrm{cel} / \mathrm{mm}, 36$ a 59 meses $<15 \%$ o $<350 \mathrm{cel} / \mathrm{mm}$; $\geq 5$ años $<15 \%$ o $<200 \mathrm{cel} / \mathrm{mm})^{5,6}$. También se evaluó el estado nutricional mediante el software WHO Anthro y Anthro Plus (versión 3.2.2, Geneva, Switzerland), clasificándolos como retardo del crecimiento (Talla para la edad $z$ score $<2$ SD debajo del promedio), emaciación (peso para la talla $z$ score <2 SD debajo del promedio), bajo peso (Peso para la edad $z$ score $<$ SD debajo del promedio), bajo peso (Peso $/$ talla $^{2}$, z score $<2 D S$ ) y se consideró una alteración de malnutrición (si presenta al menos uno de los cuatro parámetros mencionados anteriormente). Se obtuvo también información acerca de la situación familiar y los cuidadores de los niños al momento de la recolección de datos. El estudio ha sido aprobado por un comité de ética.

\section{Análisis Estadístico}

Para el análisis de la información se utilizó el Software estadístico Stata v 11.0; se hizo un análisis univariado; las variables cualitativas se informaron como frecuencias y proporciones. Las cuantitativas con distribución normal se informaron como promedios con sus desviaciones estándar; de lo contrario, se calcularon la moda y la mediana.

Con respecto al estado nutricional se comparó con un grupo de pacientes al cual denominamos control pareado por sexo, edad y momento de la hospitalización, se excluyeron a los pacientes con enfermedad crónica de más 1 mes o malformaciones congénitas, y realizamos $\mathrm{OR}$ de acuerdo a la categorización del estado nutricional con su intervalo de confianza.

\section{RESULTADOS}

Entre el 2007 y 2009, 92 niños fueron atendidos con diagnóstico de VIH de los cuales 75 fueron enrolados para el estudio, 17 niños fueron excluidos de los cuales 06 por haber iniciado TARAA en otras instituciones o fueron transferidos, 03 no presentaron indicaciones de iniciar TARAA, 08 niños presentaron información incompleta en las historias clínicas. De los 75 niños enrolados el $54,6 \%$ (41/75) fueron hombres, el promedio de edad de inicio de TARAA fue 3,6 años (rango de 3 meses a 14 años) Tabla 1 . El esquema de inicio del TARAA que incluía zidovudina (o estavudina) más nelfinavir fueron $41,3 \%(31 / 75)$, zidovudina (o estavudina) más nevirapina fueron $22,7 \%(17 / 75)$, zidovudina (o estavudina) más efavirenz fueron $21,3 \%(16 / 75)$, zidovudina (o estavudina), lamivudine más lopinavir/rit fueron $13,3 \%(10 / 75)$, solo un pacientes recibió lopina$\mathrm{vir} /$ rit más atazanavir y lamivudina $1,3 \%(1 / 75)$. 04 pacientes presentaron diagnóstico de tuberculosis.

La presentación clínica de los niños previo al diagnóstico de VIH son los siguientes: el respiratorio representa el $72 \%$ (54/75), gastrointestinal $48 \%(36 / 75)$, desnutrición 20\%(15/75), hematológico $17,3 \%(13 / 75)$, dérmico $16 \%(12 / 75)$, hepático 12,0\%(09/75), neurológico 12\%(09/75), retardo psicomotor $9,3 \%(07 / 75)$, candidemia $8 \%(6 / 75)$, siendo el $28,9 \%(21 / 75)$ la asociación gastrointestinal con respiratorio del $28 \%$ $(21 / 75)$.

La mediana de la carga viral es $\log 5,14$. La carga viral mayor de $\log 5$ representa el 62,7\%(47/75) de todos los casos, de cuales el 70,2\%(33/47) se encuentran en categoría de inmunodepresión se- 
vero (chi2 de 0,08$)$ y son menores de un año el $55,3 \%(26 / 47)$.

La mediana del numero de linfocitos CD4 en sangre periférica inicial fue de $388 \mathrm{cel} / \mathrm{ml}$ con el RIQ de $150-705$, de los cuales $21 \%$ (28/75) son menores de $200 \mathrm{cel} / \mathrm{ml}$, el rango abarca de $3 \mathrm{cel} /$ $\mathrm{ml}$ hasta $2489 \mathrm{cel} / \mathrm{ml}$.

\section{Características sociales}

La mayoría de los niños que viven con VIH figuran como procedencia del distrito de Lima, la cuarta parte de las madres son mayores de 35 años, el $26,7 \%$ corresponden a más de la segunda gestación, la tercera parte de los niños HIV son huérfanos de padres o madres y siendo la tercera parte de los niños con VIH con un responsable de su cuidado diferente al padre o madre, el 16\% de los niños se encuentran en albergue, tabla $\mathrm{N}^{\circ} 02$.

\section{Estado nutricional}

El porcentaje de los niños con VIH con alguna grado de desnutrición considerando solo

Tabla 1. Características demográficas y de laboratorio de niños infectados con VIH que inician TARAA, INSN, 2007-2009.

\begin{tabular}{|c|c|}
\hline \multicolumn{2}{|c|}{$\begin{array}{l}\text { Características demográficas y de laboratorio de niños infectados con VIH que } \\
\text { inician TARAA, INSN, 2007-2009. }\end{array}$} \\
\hline$N^{\circ}$ niños & 75 \\
\hline Edad Inicio TARAA, Mediana & 2 \\
\hline RIQ & $0.8-5$ \\
\hline \multicolumn{2}{|l|}{ Sexo } \\
\hline Hombres & $54.6 \%(41 / 75)$ \\
\hline Mujeres & $45.3 \%(34 / 75)$ \\
\hline \multicolumn{2}{|l|}{ Estadio inmunológico } \\
\hline Depresión severa $(C D 4<25 \%)$ & $68.0 \%(51 / 75)$ \\
\hline Indicación de TARAA & $78.6 \%(59 / 75)$ \\
\hline \multicolumn{2}{|l|}{ Carga viral } \\
\hline Mediana $(\log )$ & $139200(5.14)$ \\
\hline RIQ $\_l o g$ & 4.6-5.6 \\
\hline \multicolumn{2}{|l|}{ Algún indicador de malnutrición } \\
\hline Peso bajo & $66.7 \%(50 / 75)$ \\
\hline Retraso del crecimiento & $60.0 \%(45 / 75)$ \\
\hline Emaciación & $60.0 \%(30 / 50)$ \\
\hline Algún grado & $69.3 \%(52 / 75)$ \\
\hline
\end{tabular}

Nota: RIQ = rango interquartil. Chi $x 2$ test (Fisher's exact test) para variables discretas o Test Wilcoxon rank-sum o $t$ test fue usado para variables continuas. $p$ Valor $<0,05$.

${ }^{*}$ Para niños menores de 1 años $\mathrm{p}=0,032,{ }^{* *}$ Profilaxis trasmisión vertical con antirretroviral †Peso bajo - peso para la edad $z$ score $<2$ DS debajo del promedio.

${ }^{*}$ Retraso del Crecimiento-Talla para la edad $z$ score $<2$ DS debajo del promedio.

$\neq$ Emaciación-peso para la talla $z$ score $<2$ DS debajo de la mediana, solo calculado para la edad de $0-5$.

$\S$ Indicador de malnutrición, incluye, bajo peso, retrase de crecimiento o emaciación (definido arriba). 
algún parámetro del diagnóstico nutricional es de $69,3 \%(52 / 75)$, siendo el déficit nutricional de peso para la edad bajo más frecuente $66,7 \%(50 / 75)$. Encontramos un OR 10,5 de déficit nutricional de peso para la edad seguido de un OR 6,8 de emaciación, retraso del crecimiento OR 5,5, para los mayores de 5 años IMC bajo presentó un OR de 5,1, Tabla 03.

\section{DISCUSIÓN}

Si bien el uso de TARAA reduce significativamente la mortalidad en los pacientes con VIH, las familias afectadas por esta epidemia enfrentan nuevos desafíos especialmente en el ámbito social, si consideramos que la edad de las madres con VIH que trasmitieron la infección de forma vertical con VIH se concentra entre los 21 y 35 años, grupo de edad considerada como productivos por la actividad laboral y procreación en las familias.

La muerte de los padres de familia con VIH reduce la capacidad de los hogares para mantenerse por sí mismos, por lo que conlleva a la desaparición de familias e incremento de miembros en otras que en su mayoría son los abuelos o tíos, en nuestro estudio encontramos que el $14 \%$ son niños huérfanos de padre o madre, esta pérdida de un padre puede dejar a las mujeres y los niños con menos oportunidades económicas y menos control sobre la vivienda, pero la pérdida de una mujer puede resultar en falta de atención en los niños, aumento de la desnutrición ${ }^{7,8}$, in-

Tabla 2. Aspectos familiares de los niños con VIH, INSN, 2007-2009

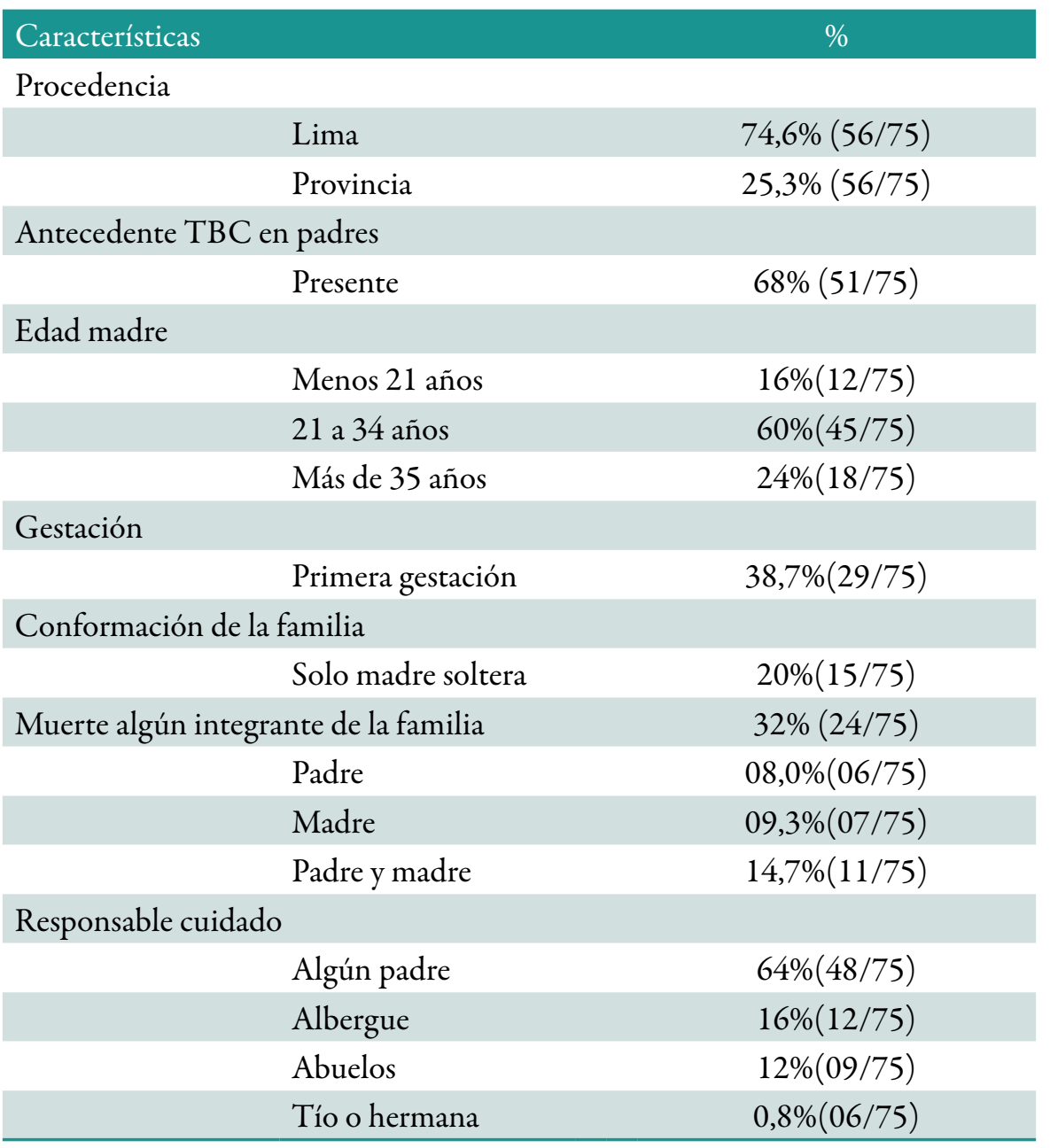


Tabla 3. Estado nutricional de los niños con VIH, INSN, 2007-2009

\begin{tabular}{lccccc} 
& VIH & no VIH & OR & IC & p \\
\hline Peso bajo & $66,7 \%(50 / 75)$ & $16,0 \%(12 / 75)$ & 10,5 & $4,5-25,0$ & 0,000 \\
\hline Retraso del crecimiento & $60,0 \%(45 / 75)$ & $21,3 \%(16 / 75)$ & 5,5 & $2,5-12,2$ & 0,000 \\
\hline Emaciación & $60,0 \%(30 / 50)$ & $09,3 \%(09 / 41)$ & 6,8 & $2,5-19,3$ & 0,000 \\
\hline IMC & $56,6 \%(14 / 25)$ & $20,0 \%(05 / 25)$ & 5,1 & $1,2-22,4$ & 0,008 \\
\hline Algún grado & $69,3 \%(52 / 75)$ & $26,7 \%(20 / 75)$ & 6,2 & $2,9-13,4$ & 0,000
\end{tabular}

formación valiosa e importante para mejorar la relación de ayuda con los niños y los padres o cuidadores, función primordial del personal de enfermería que tiene mayor contacto con las familias de los pacientes.

Debemos tener presente que no solo la pérdida de algún integrante de la familia deja al desamparo a los niños con VIH, en nuestro estudio hallamos que la tercera parte de los niños estudiados no están al cuidado de padres, ocupándose de ellos sus familiares, ocasionando en ellos mayor carga familiar o estigmatización dentro del mismo hogar, como consecuencia muchos niños terminan en orfanatos.

La desnutrición al momento del diagnóstico de VIH de los niños fue de 69,3\% considerando solo algún parámetro del diagnóstico nutricional, siendo esto alto y corresponde al déficit nutricional del peso para la edad con el $66,7 \%$, valores parecidos lo hallamos en países en vías de desarrollo como el nuestro ${ }^{9-11}$; esto representa el riesgo de 10,5 veces más de tener un déficit del peso para la edad en niños con VIH en comparación con los niños hospitalizados no infectados con VIH, lo que conllevaría a la larga la disminución de la supervivencia según otros estudios ${ }^{12,13}$. Por lo que podemos decir que la pérdida de peso para la edad es uno de los parámetros antropométricos y nutricionales más afectados en los pacientes con VIH, por lo que sugerimos que el personal de salud especialmente las enfermeras deben valorar y conocer este indicador antropométrico de forma periódica con objetivos espe- cíficos en la atención de los niños como parte de evaluación integral de estos pacientes.

Otro tema no menos importante es la situación de los adolescentes que viven en hogares de familiares $\mathrm{u}$ orfanatos por la dificultad en la integración social, así como la dificultad de encontrar vivienda y su inserción laboral, por lo que sugerimos elaborar un plan de apoyo multisectorial de apoyo a los niños y adolescentes con evaluaciones permanentes frente a los nuevos desafíos en el contexto socio económicos y cultural, iguales recomendaciones lo encontramos en otros estu$\operatorname{dios}^{14}$.

La detección de la infección por VIH en el niño puede hacerse en la madre durante el embarazo $^{15-17}$. Sin embargo reporte del MINSA en el 2010 mencionó que existe hasta un 33\% de embarazos de madre con VIH no detentados ${ }^{18}$ por lo que sugerimos mayor atención en las infecciones respiratorias y diarreicas $\mathrm{u}$ otra condición clínica con evolución inadecuada y considerarlas como sospechosos de infectados con VIH, así brindar TARAA de manera oportuna evitando las complicaciones producto de inmunodepresión crónica.

La mayoría de los niños con VIH presentó como antecedente la presencia de algún padre o algún familiar con tuberculosis, esto indica exposición a esta enfermedad, por lo que sugerimos mejorar el diagnostico de tuberculosis mediante las pruebas de laboratorio más sensible y especifico y considerar también la posibilidad de reactivación 
ante un eventual deterioro clínico o inmunológico; condiciones importantes que el personal de enfermería debe considerar en la diseminación de la tuberculosis a nivel hospitalario y adecuar medidas de prevención de la transmisión del bacilo en los niños enfermos con VIH y en el personal de la salud quien lo atiende.

\section{CONCLUSIÓN}

- Existe un riesgo elevado de déficit de estado nutricional especialmente en el peso para la edad en los niños con VIH al momento de su diagnóstico.

- Muchos niños con VIH presentan nuevas formas de convivencias bajo el cuidado de sus familiares u orfanatos que lo hacen propensos a la dificultad en la inserción social.

- Sugerimos valorar el estado nutricional de los niños con VIH e intervenir en su mejoría con programas multisectoriales adecuados que deben ayudar también a su integración social.

\section{Limitaciones}

La naturaleza de nuestro estudio limita la inferencia acerca de la causalidad y las direcciones de las asociaciones.

La falta de información específica sobre el no participante

\section{Fuentes de financiamiento}

Este trabajo ha sido autofinanciado por los autores.

\section{Conflictos de interés}

Los autores declaran no tener conflictos de interés en la publicación de este artículo.

\section{Agradecimientos}

Al personal de salud del servicio de infectologia del INSN de donde se obtuvieron la información.

\section{BIBLIOGRAFÍA}

1. Update: acquired immunodeficiency syndromeUnited States, 1994. MMWR Morb Mortal Wkly Rep 1995;44:64-7.

2. Cooper ER, Charurat M, Mofenson L, et al. 2002 Combination antiretroviral strategies for the treatment of pregnant HIV-1-infected women and prevention of perinatal HIV-1 transmission. J Acquir Immune Defic Syndr;29:484-94.

3. Ministerio de Salud., 1996. Doctrina, normas y procedimientos para el control de las ETS y el SIDA en el Perú. Programa de Control de Enfermedades de Transmisión Sexual y SIDA (PROCETSS). Lima.

4. Ministerio de Salud Perú. ; 2004. Lima Peru. Ministerio de Salud. Norma Técnica para el TARGA en adultos infectados por el VIH. Lima: MINSA. NT No 004-MINSA/DGSP-V.02.

5. Tlusty L, Hlouskova Z, Kohn R, Daum S, Stiksa J. 1966. [Pulmonary diffusion and its constituents $(\mathrm{D}-\mathrm{m}$ and $\mathrm{V}-\mathrm{c})$ in children and young adults after interstitial pneumonia]. Cas Lek Cesk;105:145-8.

6. Schneider E, Whitmore S, Glynn KM, Dominguez K, Mitsch A, McKenna MT. 2008. Revised surveillance case definitions for HIV infection among adults, adolescents, and children aged $<18$ months and for HIV infection and AIDS among children aged 18 months to $<13$ years-United States, 2008. MMWR Recomm Rep;57:1-12.

7. Bunn J, Thindwa M, Kerac M. 2009. Features associated with underlying HIV infection in severe acute childhood malnutrition: a cross sectional study. Malawi Med J; 21:108-12.

8. Nalwoga A, Maher D, Todd J, Karabarinde A, Biraro S, Grosskurth H. Nutritional status of children living in a community with high HIV prevalence in rural Uganda: a cross-sectional population-based survey. Trop Med Int Health;15:414-22.

9. Lodha R, Singhal T, Jain Y, Kabra SK, Seth P, Seth V. 2000. Pediatric HIV infection in a ter- 
tiary care center in North India: early impressions. Indian Pediatr;37:982-6.

10. Bustamante G GA, Elorza Parra M, Cornejo Ochoa W. Características clínicas de niños infectados por VIH atendidos en un hospital universitario en Medellín, Colombia, 1997-2005. IATREIA. 2007;20(4):354-61.

11. Velasco CA JAEnyaenVpctv, Universidad del Valle-Colombia 2005. J Pediatr Gatroenterol Nutr. 2005;41:508-509.

12. Fergusson P, Chinkhumba J, Grijalva-Eternod C, Banda T, Mkangama C, Tomkins A. 2009. Nutritional recovery in HIV-infected and HIVuninfected children with severe acute malnutrition. Arch Dis Child; 94:512-6.

13. Magadi MA. 2011. Household and community HIV/AIDS status and child malnutrition in sub-Saharan Africa: evidence from the demographic and health surveys. Soc Sci Med;73:436-46.

14. Abebe T, Aase A. Children, AIDS and the politics of orphan care in Ethiopia: the extended family revisited. Soc Sci Med 2007;64:2058-69.

15. Mofenson LM. U.S. Public Health Service Task Force recommendations for use of antiretroviral drugs in pregnant HIV-1-infected women for maternal health and interventions to reduce perinatal HIV-1 transmission in the United States. MMWR Recomm Rep 2002;51:1-38; quiz CE1-4.

16. Nichols SA, Bhatta MP, Lewis J, Vermund SH. 2002. Prenatal HIV counseling, testing, and antiretroviral prophylaxis by obstetric and family medicine providers in Alabama. Am J Med Sci;324:305-9.

17. Padua E, Almeida C, Nunes B, et al. 2009. Assessment of mother-to-child HIV-1 and HIV-2 transmission: an AIDS reference laboratory collaborative study. HIV Med;10:182-90.

18. Perú MdS, Dirección General de Epidemiologia. 2008. "Informe Nacional sobre los Progresos Realizado en la Aplicación el UNGASS Peridod :Enero - Diciembre 2010 .Lima: Marzo 2010. Consulta: 25 Enero 2011. En: http://www. unaids.org.
Correspondencia: Edwin Miranda e-mail: emirandach@hotmail.com 